it $10-73 t$

\title{
LA-5903-MS
}

Informal Report

UC-32 and UC-34

Reporting Date: March 1975

Issued: March 1975

72375

\section{Monte Carlo Code Development in Los Alamos}

\author{
by
}

L. L. Carter

E. D. Cashwell

C. J. Everett

C. A. Forest

R. G. Schrandt

W. M. Taylor

W. L. Thompson

G. D. Turner

\section{MASTER}


In the interest of prompt distribution, this report was not edited by the Technical Information staff.

Printed in the United Statee of America. Avoilable from National Technical Intormation Service

U S Department of Commerce 5285 Port Roral Road Springfield, VA 22151

Price: Printed Copy S4.00 Microtiche $\$ 2.25$

Thin revort was orepared an an eccount of work spongent

bi the thited sieter Gevernoment. Neither the thiend Stated

minintration, not ans of their enployees, nor any of thelr cen.

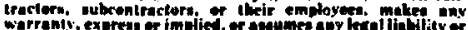

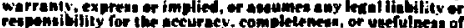

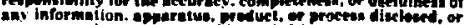

repreente that ito use wewhe met infriste privately owined

rifinte. 


\section{MONTE CARLO CODE DEVELOPMENT IN LOS ALAMOS}

by

L. L. Carter, E. D. Cashwell, C. J. Everett, C. A. Forest

R. G. Schrandt, W. M. Taylor, W. L. Thompson, and G. D. Turner

\section{ABSTRACT}

The present status of Monte Carlo code development at Los Alamos Scientific Laboratory is discussed. A brief summary is given of several of the nost important neutron, photon, and electron transport codes.

\section{INTRODUCTION}

Beginning with the ideas of von Neumann, Fermi, Ulam, and others after WorId War II when Monte Carlo emerged as a recognized discipline, Los Alamos has carried on a progran of Monte Carlo code development. The value of this method in treating complicated particle transport problems was clearly demonstrated very early in the game, and Monte Carlo played an important role in weapons development and in many other programs at the Los Alamos Scientific Laboratory. $^{1}$

In the following remarks, some of the most important Monte Carlc codes at LASL will be described briefly. All of them are under constant development in order to meet the increased demands upon them, both as to the difficulty of the problems they are asked to solve and with respect to the task of handling the vast amnunt of cross-section data now available. With one exception, the neutron and photon codes considered here use a continuous energy treatment of the cross sections. Pointwise data are provided at discrete energies, with interpolation employed in between. The cross sections are read into the codes in considerable detail in an attempt to use the information with no significant approximations or distortions. This puts a considerable burden on the storage capacity of the computer, especially in view of the increasing size of nuclear data compilations such as the Evaluated Nuclear Data Files (ENDF).

The codes described below all have the same three-dimensional geometry package. ${ }^{2}$ Each has the capability of handling an arbitrary configuration of materials in regions which are bounded by firstand second-degree surfaces, as well as some fourthdegree surfaces (elliptical tori). ${ }^{3}$ The language used in all of the following programs is FORTRAN IV.

\section{MCN - A NEUTRON MONTE CARLO CODE}

This $^{2}$ is our standard neutron transport code and it has formed the basis for a number of other codes, some of which will be mentioned $h+r e$. A great deal of work has gone into making this program as general and versatile as possible, while at the same time keeping it sinjit io use. It is the latest of a series of Monte Cario neutron codes at Los Alanos, the first of whichi was described by $R$. Johnston. 4

The pointwise cross sections used in MCN at present are from the nucleat data compilations of Howerton's group at LLL, from ENDF, or from the British (AWRE). The ENDF data are processed for use in MCN by D. R. Harris' group (Group T-2) at LASL. Pointwlse data are provided to us with the resonance treatment included, data may be thinned to prescribed

\section{NOTICE}

This report was prepared as an account of work sponsored by the United States Government. Neither the United States nor the United States Energy Research and Development Administration, not any of their employees, nor any of their contractors, subcontractors, or their employees, makes any warranty, express or implied, or assumes any legal linbility of responsibility for the accuracy, completeness or usefulness of any information, apparatus, product or process disclosed any information, apparatus produc , represents that its use would not intringe privately owned rights. 
tolerances, and in some cases interpolated data may be added in order to permit accurati linear interpolation in MCN. In particular, angular data may te excessive in some evaluations, arid by thinning, storage requirements may be reduced considerably.

Included in the code are standard variancereducing texhniques, which are optional. These include particle splitting and Russian roulette, path-length stretching, and machinery for biasing the source with respect to both energy and angle of emission. Provision is made for forcing collisions in designated cells; obtaining flux estinates at point detectors, and for calculating reactions in small regions by using track-1ength estimators.

MCN employs an energy-dependent fission routine, with pre-fission neutrons emitted in an evaporation spectrum and the remaining neutrons in a fission spectrum. Both types of spectra depend upon the energy of the incoming neutron.

The code includes a neutron thernalization routine employing the free gas model. Relow a thermal cut-i.. energy, the lighter atoms such as hydrogen and deuterium are assumed to be in thermal motion, with a Maxwellian distribution of velocities determined by the thermal temperature of the region. $1, \cdot \cdot h$ geometric cell of the problem has its own thermal temperature which may be given as a function of time--that is, these temperatures may be specified at discrete times $t_{i}$, with linear interpolation employed to $y$ ield the thermal temperature at time $t$. Scattering from the light nuclei includes the effect of the thermal motion. For nuclei not belonging to this select group of light atoms, and for energies in the thermal rarge, elastic scattering is assumed to occur isotropically in the laboratory system with no energy loss.

The standard output of MCN includes two-way currents as a function of energy, time, and angle with the normal, across any subset of bounding surfaces in the problem. Fluxes at any set of bounding surfaces are available as a function of time and energy. Similarly, the flux at designated points and the average flux in a cell (track length per unis volume) are standard tailies. Reactions such as fissions or absorptions may be obtained in a subset of the geometric cells. In addition, particles may be flagged when they cross specified surfaces or inter designated cells, and the contri- butions of these flagged particles to certain of the tallies above are listed separately. All quantities printed out have their relative errors listed also-here the relative error is defined as the ratio of one standard deviation in the sample mean to the sample mean.

\section{I11. MCNA - A NEUTRON ADJOINT CODE}

The code MCNA, ${ }^{5,6}$ a companion code to $M C N$, was set up to sample from the adjoint neutron transport equation. The identical cross sections and scattering models are used by both the forward and adjoint codes. This feature is often used to check for errors by comparing forward and adjoint results for standard test problems.

The principal use of MCNA at Los Alamos has been in computing F-numbers, i.e., in computing reaction rates in some portion of a system as a func$t$ ion of the neutron source distribution incident upon the system. The computation is typically carried out in a coupled mode to optimize the Monte Carlo. A short forward calculation is first made with a "typical" incident neutron source in order to compute the energy-dependent fIux in each region of the system. These flux estimates are then used to bias the selection of the emerging energy at collision events in the adjoint calculation by numerically approximating the near-optimal adjoint density function

$$
f\left(E ; E^{\prime}\right)_{k}=\frac{\phi(E)_{k} \Sigma\left(E \rightarrow E^{\prime}\right)_{k}}{\int \phi\left(E^{\prime \prime}\right)_{k} \Sigma\left(E^{-\infty}+E^{\prime}\right)_{k} d E^{-\prime}},
$$

where $\phi(E)_{k}$ is the energy-dependent flux averaged over the cell volume denoted by $k$ and $\Sigma\left(E \rightarrow E^{\prime}\right)_{k}$ is the cross section for an energy transfer from $E$ to $E^{\prime}$ at a collision. The "particle" weight is adjusted at each collision in the calculation to obtain unbiased results.

\section{MCG - A GAMMA-RAY TRANSPORT CODE}

We shall give a brief description of $\mathrm{ASC}^{7}{ }^{7}$ a Monte Carlo program for transporting gamna rays which has been in existence for many years. This code has a number of features in common with MCN, such as the geometry package, the variance-reduction 
techniques, and some of the output. Photon interaction cross sections from the LLL 1 ibrary ${ }^{8}$ are used.

Since this program is designed to transport high-energy photons, it considers only the following reactions:

(1) Compton Scattering - Sampling from the Klein-Nishina scattering law is accomplished very efficiently through the use of simple but accurate fits to the inverse of the distribution function 9,10 .

(2) Pair Production - When an electron-positron pair is produced by a gamma ray, the difference betheen the energy of the incoming gamma ray and the resulting anrihilation radiation is deposited locally at the sițe of collision. The two $0.511-\mathrm{MeV}$ photons emitted are assumed to be emitted isotropically at the site of collision and are transported further.

(3) Photoelectric Effect - This reaction is treated as absorption, resulting in the removal of the photon with its energy deposited locally.

The current and flux tallies described in MCN are also standard in MCC. Information about the energy deposited in each cell of the problem is automatically listed. The energy deposited due to Compton collisions, pair production, and photoelectric absorption is listed separately for each type of reaction, along with the total energy deposited per cell.

\section{v. MCP - A GENERAL MONTE CARLO PHOTON CODE}

The gamma code MCG is not designed to deal with low-energy photons, say in the range $1-50 \mathrm{keV}$ for some materials. Fluorescence and coherent scattering are ignored, and incoherent scattering is assumed to be governed by the Klien-Nishina cross section for free electrons.

The Honte Carlo code $M C P^{7}$ corrects some of the deficiencies in MCG. Designed to handle photons of energies $1 \mathrm{keV}$ to $100 \mathrm{MeV}$, MCP provides for fluorescent emission and modifies the Thomson and KleinNishina differential cross sections by scattering factors which take binding effects into account. With the exception of a more sophisticated collision routine, the code MCP has much in common with MCG, containing the same geometry routine, variance-reducing techniques, and output. The distinctive features of the collision routine are summarized:

(1) The cross sections in the compilation of Storm and 1 srael 11 are used, with $\log -10 \mathrm{~g}$ interpolation, on the energy range 0.001-100 MeV.

(2) lncoherent scattering is governed by the function

$$
K(\alpha, \mu) I(z, v) d \mu,
$$

where $K(\alpha, \mu)$ is the Klein-Nishina differential cross section and $I(2, v)$ is a form factor correcting for bound effects. Here $\alpha$ is the incoming photon energy, $z$ is the atomic number of the scattering atom, $\mu$ is the cosine of the scattering angle, and $v$ is proportional to the momentum transfer to the electron. Sampling the incoherent scattering density is effected by a rejection technique, using the rapid sampling scheme ${ }^{10}$ for the KleinNishina distribution described above for the code MCG.

(3) The coherent scattering density is proportional to the function

$$
C^{2}(z, \nu) T(\mu) d \mu
$$

where $C(Z, v)$ is a form factor modifying the energy independent Thomson cross section $T(\mu) \sim\left(1+\mu^{2}\right)$. A rejection schene is also used to sample the coherent scattering.

Although the collision routine is much more complex in this code than it is in MCG, the running times are not greatly different for all but the simplest geometries.

\section{MCNG - A COMBINED NEUTRON-GAMMA RAY TRANSPORT CODE}

This Monte Carlo code ${ }^{12}$ has the capability of transporting neutrons with the code $\mathrm{MCN}$, producing gammas from neutron reactions by means of stored gamona production cross sections, and transporting ganma rays with the code MCG. It is designed to replace the separate codes MCN and MCG, and it may be run in three modes: (1) mode 0 - MCN only;

(2) mode 1 - MCN and MCG (combined mode); and

(3) mode 2 - MCG only.

When run in the combined mode 1 , the code provides for the study of total heating or energy 
deposition in the medium by neutrons, photons, and products of neutron reactions. In a specific neutron reaction, the conservation of energy requires that

$$
k+Q=H+N^{-}+I,
$$

where $k$ is the kinetic energy of the neutron, $Q$ is the Q-value of the reaction and depends only on the rest energies of the material particles involved, $H$ is the total kinetic energy of the charged particles or nuclear products resulting, $N^{-}$stands for the sum of the kinetic energies of all outcoming neutrons, and $\Gamma^{-}$is the total energy of the gammas enitted. Partly because of the complexity of gamma production data and the existence of cross sections for materials consisting of natural mixtures of isotopes for which the component cross-section data are unknown, the code MCNG computes the heating per neutron collision,

$$
\overline{\mathrm{H}}=k+\overline{\mathrm{Q}}-\overline{\mathrm{N}}^{\bullet}-\overline{\mathrm{T}} \text {. }
$$

$\overline{\mathrm{H}}$ is the expected nuclear product heating per neutron collision and $\bar{Q}, \bar{N}^{-}, \bar{T}$ are the corresponding expectations per collision of the rest energy change, of the neutron energy emitted, and of the gamma energy produced. An additional reason for obtaining the charged particle heating per collision is to reduce statistical fluctuations inherent in sampling the individual reactions. The expected heating per collision, $\bar{H}$, is listed on the same en srgy grid that the total reaction cross sections use.

Liberated photons may produce further heating from pair production, Compton scattering, and photoelectric absorption. This photon energy deposition is also given as the expected heating per photon collision. Similarly, the product neutrons will give rise to additional heating from further collisions. In transporting neutrons by MCN, the gammas produced from collisions with the medium are stored on tape, and they form a source which is processed by MCG at the end of the neutron cycle. The coda is designed to cycle between these two codes until the required statistical accuracy is attained. The heating tallies give the energy deposition per starting neutron. In addition to collision tallies, provision is made in the code to obtain heating in small regions by track length tallies.

Currently the gamma production cross sections are used in multigroup form ( 30 neutron groups and 12 garma groups), even though our transport codes use a continuous energy dependence on cross sections. This has been a matter of convenience, since some multigroup data existed when the code was set up. Cur present plans call for the ganma production cross sections to be given on the same energy points our neutron cross sections use, with the gamma output spectra to be given in much finer detail.

\section{VIJ. MCK - A MONTE CARLO CRITICALITY CODE}

One of the codes which is a modification of MCN--with essentially the same input, collision routine, biasing techniques, and general geometry package-is the code MCK. This program is an iterative scheme, similar in many respects to the code KENO, ${ }^{13}$ for finding the multiplication in a static configuration containing fissile materials. From a given fission source, the code transports the neutrons, creating a new fission source. The procedure is iterative, using the latest distribution as the source for a new cycle until convergence is attained. The multipljcation constant $k_{\text {eff }}$ is estimated from the ratio of the neutron population in the $(n+1)^{\text {st }}$ generation to that in the $\mathrm{n}^{\text {th }}$ generation.

The source is self-corrective in the sense that if the user decides to use a source of $\mathrm{N}$ particles, then the code generates a new source of approximately $\mathrm{N}$ neutrons. Of course, the total weight started from the source at the beginning of each cycle remains constant. Since the code is adapted from MCN, it contains the thermalization routine as well as the energy-dependent fission treatment.

Simple to use and versatile because of its general geometry, this program has been applied to a wide range of problems, e.g., in the design of very complex reactors ${ }^{14}$ as well as in safety calculations.

\section{MCMG - MULTIGROUP MONTE CARLO}

The continuous energy Monte Carlo codes have Monte Carlo effort. However, there are some applications for which our present codes are somewhat 
limited. Consequently we have developed a multigroup code with three general problem areas in mind: (1) the treatment of adjoint problems not amenable to solution with MCNA, e.g., coupled neutron and neutron-induced gamma-ray transport problems;

(2) calculations by both multigroup Monte Carlo and multigroup discrete ordinates methods, using identical cross-section sets; (3) the simulation of particle transport with relatively simple crosssection input.

The programming of the multigroup code MCMG is based on our combined neutron-gamma code MCNG. Most of the input data, the geometry package, the biasing schemes, the majority of the output formats, and many of the control cards are identical to their counterparts in MCNG. The code permits the multigroup cross sections to be input in a variety of standard formats. It will operate in either the forward or adjoint mode.

Various methods have been suggested in the literature for treating the angular scattering distribution after a group-to-group scattering event. Three options are provided in MCMG. The first uses the first $N$ Legendre moments to construct a step function with $N$ - 1 non-zero steps which conserves (in some cases, approximately) these $\mathrm{N}$ moments. The area under each non-zero step is chosen to be $1 /(N-1)$, so that the $\mu_{i}$-endpoints of the non-zero steps are determined from the $N$ moments. The second option is the method used in the MORSE code which allows the scattering to, occur at discrete angles. The third option samples the scattering angle from a density function that is proportional to the absolute value of a truncated Legendre series. The last option can lead to negative weights and, from a Monte Carlo standpoint, is not usually a desirable mode. It is included as an option since it is a close analog to the discrete ordinates treatment.

IX. MCG: - A COUPLED ELECTRON-PHOTON TRANSPORT CONE One of the newer members of our family of Monte Carlo codes is an electron-photon transport code containing the general three-dimensional geometry package. This program goes to considerable lengths to include the physics of electron-photon interactions and the transport of both types of radiation. In the primary-photon mode, the effect of secondary electrons can be studied definitively.
Cases in which secondary electrons are important include (1) response function calculations of detector systems if the range of the typical secondary electron is comparable to the dimensions of the system; (2) gama-ray shielding calculations if bremsstrahlung is important--in a 6 mfp lead shield irradiated by $10-\mathrm{MeV}$ photons, bremsstrahlung increases the Goldstein-Wilkins exposure buiidup factor by $60 \%$ and the dose albedo factor by $140 \%$; and (3) gamma-ray heating problems if local energy deposition is a poor assumption (because the energy is transported by secondary electrons and bremsstrahlung). In the primary-electron mode, the code is useful in the study of electron transport and the production of bremsstrahiung. Applications include verification of experimental results from high-energy electron accelerators, laser fusion calculations, and energy deposition in tissue and bone for medical studies.

MCGE $^{15}$ treats the physics of electron and pheton transport, including the generation of bremsstrahlung radiation, in considerable detail. The electron portion of the code is based on the procedures used by Berger and Seltzer in ETRAN ${ }^{16}$ but includes several modifications and refinements not found in ETRAN-most apparent is the generalized geometiy, mixedmedia capability of MCGE.

Photon histories are followed with standard collision-by-collision Monte Carlo methods, accountj.ng for Compton scattering, pair production, and photoelectric absorption. Photoelectric absorption is considered explicitly in order to include the effect of the photoelectron. Pair production in MCGE refers only to the absorption of a photon and the subsequent production of an electron-positron pair. Annihilation is a completely separate event, occurring only at the end of the positron's path (path and range are not equated) and not at the point where the electron-positron pair was produced. If the positron escapes from the system, the annihilation quanta are not produced.

Histories of electrons (and positrons) are folloked in MCGF by using multiple-scattering theories to describe the electron's behavior over a small increment of its path, not collision-by-collision since tens of thousands of collisions may be associated with each history. The electron energy loss (including straggling effects) over an increment of 
its path is determined by sampling the appropriate thecries to account for ionization, bremsstrahlung, and the angular deflection. The production of bremsstrahlung in each increment is determined from corrected cross sections derived by using the Born approximation.

All generations of electrons and photons are accounted for in MCGE: delta rays, bremsstrahlung, characteristic $x$ rays, photoelectrons, Compton electrons, the electron-positron pairs, annihilation quanta, fluorescence radiation, and Auger elestrons. The code requires about 300000 decimal words of computer storage, and the problem of $10-\mathrm{MeV}$ photons normally incident on a 6 mfp lead alab requires about two hours of $C D C-7600$ computer time for 40000 primary photon histories.

\section{MCGB - A GAMA CODE WITH BREMSSTRAHLUNG}

The conventional gamma code MCG described earlier has been modified to include the effects of secondary electrons (i.e., bremsstrahlung) for situations where the dimensions of the system are much greater than the typical range of a secondary electron--e.g., in gamma-ray shielding calculations. MCGB includes the effects of bremsstrchlung, without going to the trouble and expense of actually following secondary electron histories, by using thick-target bremsstrahlung theory. ${ }^{17}$ only 50000 decimal words of storage are needed (much of this is taken up by the geometry package--a one-dimensional version would need only a few thousand words) and the 40000 histories of $10-\mathrm{MeV}$ gamma rays in lead require only 10 minutes of $\operatorname{CDC}-7600$ time. MCGB is basically a simple Monte Carlo code. Compton scattering, pair production, and photoelectric absorption are accounted for using the conventions of MCC (photoelectric absorption is not "weightedout," however, since the photoelectron is needed for bremsstrahlung production). The energy and direction of the Compton recoil electrons are easily found from energy and momentum relations. The photoelectron is assumed to be produced straight ahead (a very good assumption if the energy of the electron is greater than $1 \mathrm{MeV}$ ) with energy equal to the energy of the absorbed photon, neglecting the binding energy. The energy distribution of the members in the electron-positron pair is assumed to be equally split, with each member having kinetic energy equal to $\left(k-2 m_{0} c^{2}\right) / 2, k$ being the energy of the incident photon. The emission angle of the pair is assumed to be $m_{0} c^{2} / T$ radians, where $T$ is the kinetic energy of the electron or positron.

\section{REFERENCES}

1. E. D. Cashwell and C. J. Everett, A Practical Manual on the Monte Carlo Method for Random Wa1k Problems (Perganon Press, New York, 1959).

2. E. D. Cashwe11, J. R. Neergaard, W. M. Taylor, and G. D. Turner, "MCN: A Neutron Monte Carlo Code," Los Alamos Scientific Laboratory repcrt LA-4751 (1972).

3. E. D. Cashwel1 and C. J. Everett, "Intersection of a Ray with a Surface of Third or Fourth Degree," Los Alamos Scientific Laboratory report LA-4299 (1969).

4. R. R. Johnston, "A General Monte Carlo Neutronics Code," Los Mlamos Scientific Laboratory report LA-2856-MS (1963).

5. L. L. Carter and N. J. McCormick, "Coupled Sampling with the Monte Carlo Method in Neutron Transport Calculations," Nucl. Sci. Eng., 39, 296-310 (1970).

6. L. L. Carter, "MCNA: A Computer Program to Solve the Adjoint Neutron Transport Equation by Coupled Sampling with the Monte Carlo Method," Los Alamos Scientific Laboratory report LA-4488 (1971).

7. E. D. Casine11, J. R. Neergaard, C. J. Everett, R. C. Schrandt, W. M. Taylor, and G. D. Turner, "Monte Carlo Photon Codes: MCG and MCP," Los Alanos Scientific Laboratory report LA-5157-MS (1973).

8. E. F. Plechaty and J. R. Terrall, "Photon Cross Sections $1 \mathrm{keV}$ to $100 \mathrm{MeV}$," Lawrence Livermore Laboratory report UCRL-50400, Vol. VI (1968).

9. C. J. Everett and E. D. Cashwe 11, "Approximation for the Inverse of the Klein-Nishina Probability Distribution Function," Los Alamos Scientific Laboratory report LA-4448 (1970).

10. C. J. Everett, E. D. Cashwe11, and G. D. Turner, "A New Method of Sampling the Klein-Nishina Probability Distribution for All Incident Photon Fnergies Above $1 \mathrm{keV}, "$ Los Alamos Scientific Laboratory report LA-4663 (1971).

11. E. Storm and H. I. Israel, "Photon Cross Sections from $1 \mathrm{keV}$ to $100 \mathrm{MeV}$ for Elements $Z=1$ to $z=100, "$ Nuclear Data Tables 7, 565-681 (1970).

12. Report in preparation.

13. G. E. Whitesides and N. F. Cross, "KENO - A Multigroup Monte Carlo Criticality Program," Union Carbide Corporation, Nuclear Division, Report CTC-5, Oak Ridge, Tennessee (1969). 
14. C. W. Watson and E. D. Cashwe11, Monte Carlo Development and Applications in the Los Alanos Nuclear Rocket Program," Proceedings of the Fourth International Conference on Reactor Shielding, Vol. 1, Paris (Oct. 1972).

15. W. L. Thompson, "Gamma-Ray and Electron Transport by Monte Car1o," Doctoral Dissertation. University of Virginia, Charlottesville, Va. (February 1974).
16. M. J. Berger and S. M. SeItzer, "Electron and Photon Transport Prograns," National Bureau of Standards Report 9837 (June 1968).

17. C. R. Emigh, "Thick Target Brensstrahlung Theory," Los Alamos Scientific Laboratory report LA-4097-MS (March 1970). 